UUITP-23/11

\title{
Supergraphs and the cubic Leigh-Strassler model
}

\author{
Joseph A. Minahan \\ Department of Physics and Astronomy \\ Uppsala University \\ Box 520 \\ SE-751 20 Uppsala, Sweden \\ joseph.minahan@fysast.uu.se
}

\begin{abstract}
We discuss supergraphs and their relation to "chiral functions" in $\mathcal{N}=4$ Super Yang-Mills. Based on the magnon dispersion relation and an explicit three-loop result of Sieg's we make an all loop conjecture for the rational contributions of certain classes of supergraphs. We then apply superspace techniques to the "cubic" branch of Leigh-Strassler $\mathcal{N}=1$ superconformal theories. We show that there are order $2^{L} / L$ single trace operators of length $L$ which have zero anomalous dimensions to all loop order in the planar limit. We then compute the anomalous dimensions for another class of single trace operators we call one-pair states. Using the conjecture we can find a simple expression for the rational part of the anomalous dimension which we argue is valid at least up to and including five-loop order. Based on an explicit computation we can compute the anomalous dimension for these operators to four loops.
\end{abstract}




\section{Contents}

1 Introduction $\quad 1$

2 Superspace for composite operators $\quad 4$

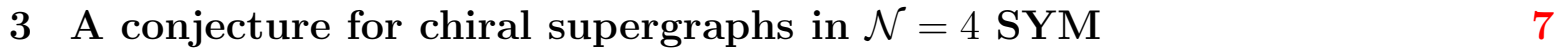

4 Application to the cubic Leigh-Strassler model $\quad 10$

4.1 The cubic model and its corrections to the coupling . . . . . . . . . . . 10

4.2 Planar protected states . . . . . . . . . . . . . . . . . . . . . . . . . . . . . . . . . 12

4.3 The one-pair states . . . . . . . . . . . . . . . . . . . . . 12

4.4 Multi-paired states . . . . . . . . . . . . . . . . . . 16

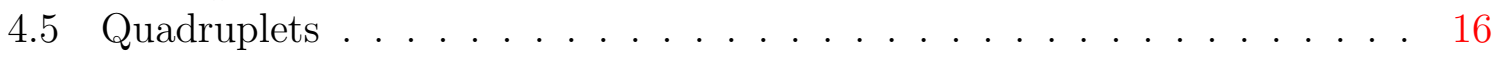

4.6 A finite Hagedorn temperature at strong coupling? . . . . . . . . . . . . 17

$\begin{array}{llr}5 & \text { Discussion } & 18\end{array}$

$\begin{array}{lr}\text { A Appendix } & 20\end{array}$

\section{Introduction}

Integrability has proven to be a powerful tool in computing the spectrum of operators in highly superconformal gauge theories (see [1] for a comprehensive review.) The most studied theory is of course $\mathcal{N}=4$ Super Yang-Mills (SYM). A while ago, Leigh and Strassler showed that $\mathcal{N}=4 \mathrm{SYM}$ has three independent deformations that preserve $\mathcal{N}=1$ superconformal symmetry [2]. The Leigh-Strassler superpotential can be written as

$$
W=\kappa\left(\operatorname{Tr}(X Y Z-q X Z Y)+\frac{h}{3}\left(\operatorname{Tr} X^{3}+\operatorname{Tr} Y^{3}+\operatorname{Tr} Z^{3}\right)\right)
$$

By a chiral field phase rotation $\kappa$ can be chosen real. There is a further relation of the couplings to $g_{\mathrm{YM}}$, the Yang-Mills coupling, leaving a three complex dimensional space of $\mathcal{N}=1$ superconformal theories.

Leigh and Strassler's argument is essentially an existence proof for the marginal directions, but the relation of the couplings is known, at least to the first few loop orders, and in the planar limit is given by [3]

$$
2 g_{\mathrm{YM}}^{2}=\kappa \bar{\kappa}(1+q \bar{q}+h \bar{h}) .
$$

The most well known deformation is the so-called $\beta$-deformation where $h=0$ and $q=e^{-2 i \pi \beta}$ with $\beta$ real (see [4] for a review). After a field redefinition, this modifies the $\mathcal{N}=4$ superpotential to

$$
W=g_{\mathrm{YM}} \operatorname{Tr}(X[Y, Z]) \rightarrow g_{\mathrm{YM}} \operatorname{Tr}\left(e^{i \pi \beta} X Y Z-e^{-i \pi \beta} X Z Y\right) .
$$

Staying in the planar limit, the relation in (1.2) is exact [5,6]. Furthermore, the computation for the spectrum of local operators is an integrable problem [7-10]. In [8] it 
was shown at the one-loop level that the corresponding Bethe equations are the same as $\mathcal{N}=4$ except for a $\beta$-dependent shift. This was extended to all loops in [9]. The supergravity dual for the $\beta$-deformed theory is known [11] and its world-sheet theory has been shown to be classically integrable, even though it is not a coset $[10,12]$.

For a general deformation it appears that integrability might be lost. Even at the one-loop level, for complex $\beta$ the resulting spin-chain is a known integrable model only in the $S U(2)$ sector [8]. Moreover, the relation between the couplings in (1.2) gets higher loop corrections starting at four-loop order $[5,13,14]$.

Less attention has been paid to deformations with nonzero $h$, although interesting results have still been found. In [15-17] it has been shown that for the choice $q=$ $(1+\rho) e^{\pi i n / 3}, h=\rho e^{\pi i m / 3}$ where $\rho$ is real and $n$ and $m$ are integers, the $R$-matrix in the $S U(3)$ sector satisfies the Yang-Baxter equation. However, it was also shown that all such theories are related to the $\beta$-deformed theories via similarity transformations [15]. Another interesting branch has $q=0$ and $|h|=1$, which was shown to be integrable at the one-loop level [17] and also has been conjectured to require no higher-loop corrections to $(1.2)[18]$.

In this paper we consider a branch of Leigh-Strassler theories that is far away from the $\mathcal{N}=4$ branch. Here we let $\kappa=0$ in (1.1) while at the same time taking $h \rightarrow \infty$ such that $\widehat{h} \equiv h \kappa$ is finite. The superpotential then takes the form

$$
W=\frac{\widehat{h}}{3}\left(\operatorname{Tr} X^{3}+\operatorname{Tr} Y^{3}+\operatorname{Tr} Z^{3}\right)
$$

where $\widehat{h}$ can be chosen real. We call this the "cubic" Leigh-Strassler model ${ }^{1}$. The model has a $U(1) R$-symmetry, but unlike the $\beta$-deformed theory, it does not have an extra $U(1) \times U(1)$ global symmetry. It does have an $S_{3} \times Z_{3} \times Z_{3}$ discrete symmetry, which is a larger symmetry group than the generic superpotential in (1.1), which only has a $Z_{3} \times Z_{3}$ symmetry.

Using the relations of the couplings in (1.2), we see that for the cubic model $|\widehat{h}|^{2}=$ $2 g_{\mathrm{YM}}^{2}$ in the planar limit, at least to the lowest few loop orders. Starting at four-loops this relation gets corrected [18]. Given the nature of the superpotential in (1.4) many single trace operators composed of scalar fields do not mix with other operators. In fact, we will show that an exponentially large number, $\sim 2^{L} / L$ where $L$ is the length of the operator, receive no anomalous dimensions to any loop order in the planar limit. We will refer to such states as planar protected. This suggests that the gravity dual for this theory is stringy in nature.

This result about planar protected states relies on properties of supergraphs [19] (see [20] for a review that centers on supergraphs in $\mathcal{N}=4$ SYM.) Supergraph technology can vastly reduce the number of Feynman diagrams that appear in an anomalous dimension calculation. In the case of the $S U(2)$ sector of $\mathcal{N}=4 \mathrm{SYM}$, the one-loop diagrams in [21] are reduced to a single diagram using supergraphs. At higher loops, the heroic effort to compute the four-loop wrapping corrections in $[22,23]$ would have been wellnigh impossible without using supergraphs. Even in ABJM/ABJ theories, the four-loop calculations in $[24,25]$ to compute corrections to an unknown function of the 't Hooft parameters were simplified in [26] by using $\mathcal{N}=2$ superspace [27]. In both $\mathcal{N}=4 \mathrm{SYM}$

\footnotetext{
${ }^{1}$ Of course, the entire Leigh-Strassler moduli space is cubic. The name chosen here is intended to be a shortened version of Fermat cubic.
} 
and $\mathrm{ABJM} / \mathrm{ABJ}$, a key to the simplification is a set of finiteness conditions that can be used to discard many graphs [28].

An important property of the supergraphs is that they naturally come with factors of so-called chiral functions (to be explained in the text). The chiral functions can be read off from the "skeletal" part of the supergraph, which is the supergraph with all vector propagators and vertices removed. In the $S U(2)$ sector many of the chiral functions are equivalent and one ends up combining the different classes of supergraphs together to, say, compare a supergraph calculation with a result expected from the magnon dispersion relation [28].

In this paper we offer a conjecture that disentangles the rational contributions of certain chiral functions to the magnon dispersion relation. It is based on Sieg's explicit three-loop computation [28] of the dilation operator in the $S U(2)$ sector [29]. It proposes a natural decomposition of individual contributions which are indistinguishable in this sector, but act differently outside of it. This allows us to find an all-loop contribution for the rational part of supergraphs with a particular chiral function. This technique does not work for all chiral functions, but only for a class of them that we call connected.

The chiral functions arise out of the $\mathcal{N}=4$ superpotential, but given the all-loop contribution of supergraphs for the connected chiral functions, we can apply it to the $\mathcal{N}=1$ superconformal deformations by replacing the $\mathcal{N}=4$ chiral functions with those appropriate for the $\mathcal{N}=1$ theory. In the $\beta$-deformed case this is straightforward $[30,31]$, but for the cubic model this is more complicated because of the four-loop correction to $\widehat{h}$. The correction implies that we must include additional supergraphs in the overall computation, and depending on one's prescription, infinite counterterms. Nevertheless, we will argue that for a particular class of single trace operators the tuning of $\widehat{h}$ that keeps the cubic model superconformal also cancels out the contributions of the additional supergraphs, at least to five loops. For these states only one chiral function acts nontrivially and we use its form for the cubic model to explicitly find the anomalous dimension of these operators. Starting at six loops there are other types of these extra diagrams that we have not yet determined if they will cancel with the tuning.

While we claim that the conjecture is valid for the rational contributions, starting at four loops there are higher transcendental contributions that do not cancel out for the individual chiral functions, but do cancel once identifications are made between chiral functions [32]. Nonetheless, certain chiral functions are easier to compute than others, and one can use the $\mathcal{N}=4$ dispersion relation to extract the contribution of the more difficult to compute chiral function by directly computing the easier one. We have done this at the four-loop level [32] to find the chiral function relevant for our $\mathcal{N}=1$ theory and can thereby find the anomalous dimensions to four-loop order for this class of operators.

In section 2 we review some relevant properties of superspace diagrams. In section 3 we make the conjecture relating the rational part of superspace diagrams to the dilatation operator. In section 4 we apply the results of sections 2 and 3 to the cubic model. We first show how to take the corrections of $\widehat{h}$ into account. We then present the one-loop Hamiltonian in the chiral scalar sector, writing it as an $S U(3)$ analog of the anti-ferromagnetic Ising model. At higher loops, aside from arguing the claims of the preceding paragraphs, we also briefly discuss some other operators outside of these classes. We also discuss the possibility of a finite Hagedorn temperature at strong cou- 
pling. In section 5 we summarize our results and discuss future directions.

\section{Superspace for composite operators}

In this section we discuss the correlators of composite operators in superspace $[19,20]$. For the purposes of this discussion, a composite operator $\mathcal{O}$ in $\mathcal{N}=4 \mathrm{SYM}$ and their $\mathcal{N}=1$ deformations will mean a single trace operator containing the scalar chiral fields $X, Y$ and $Z$, which we also write as $\phi^{i}, i=1,2,3$, as well as the gaugino chiral field $W_{\alpha}=-\frac{1}{4} \bar{D}^{2} D_{\alpha} V$, where $V$ is the vector superfield. The anti-chiral fields are given by $\bar{\phi}_{i}$ and $\bar{W}_{\dot{\alpha}}$. In $\mathcal{N}=4$ language, these are the operators that make up the closed $S U(2 \mid 3)$ sector [33]. We will also consider scalar composites where only the scalar fields are present.

The building blocks of a supergraph are the propagators for the chiral and the vector fields, and the vertices. A chiral field progagator is denoted by

$$
\left\langle\phi^{i}{ }_{b}{ }_{b} \bar{\phi}_{j_{d}}^{c}\right\rangle=\overline{\bar{p}}=\delta^{i}{ }_{j} \frac{\delta^{a}{ }_{d} \delta^{c} b}{p^{2}} \delta^{4}\left(\theta_{1}-\theta_{2}\right)
$$

where the raised indices $a, c$ refer to fundamental gauge indices and the lowered indices $b, d$ refer to anti-fundamental gauge indices. Strictly speaking this is the propagator for a $U(N)$ gauge theory, but this is immaterial in the large $N$ limit. Likewise, the propagator for the vector fields is

$$
\left\langle V_{b}^{a} V_{d}^{c}\right\rangle=\widetilde{\sim} \sim=-\frac{\delta^{a}{ }_{d} \delta^{c}{ }_{b}}{p^{2}} \delta^{4}\left(\theta_{1}-\theta_{2}\right)
$$

Note that the superspace propagators have dimension -4 .

The possible vertices include the chiral vertex,

$$
V_{\phi^{i} \phi^{j} \phi^{k}}=\overbrace{\delta_{j}} i\left(G_{i j k} \delta^{a_{2}}{ }_{b_{1}} \delta_{b_{2}}^{a_{3}} \delta_{b_{3}}^{a_{1}}+G_{i k j} \delta_{b_{1}}^{a_{3}} \delta_{b_{2}}^{a_{1}} \delta^{a_{2}} b_{3}\right)
$$

where $G_{i j k}$ is a coupling that depends on the relevant theory. In the case of $\mathcal{N}=4$ SYM we have that $G_{i j k}=g_{\mathrm{YM}} \epsilon_{i j k}$. The gauge indices are numbered in the order that the fields appear in the vertex. Two of the three legs of the vertex include the square of super-derivatives $\overline{\mathrm{D}}^{2}=\epsilon^{\dot{\alpha} \dot{\beta}} \overline{\mathrm{D}}_{\dot{\alpha}} \overline{\mathrm{D}}_{\dot{\beta}}$. Since the superpotential has no mass terms, a chiral vertex cannot connect with another chiral vertex.

There is also the anti-chiral vertex

$$
V_{\bar{\phi}_{i} \bar{\phi}_{j} \bar{\phi}_{k}}=\overbrace{\theta_{j}}^{\hat{q}_{j}} i\left(G^{i j k} \delta_{b_{1}}^{a_{2}} \delta_{b_{2}}^{a_{3}} \delta_{b_{3}}^{a_{1}}+G^{i k j} \delta_{b_{1}}^{a_{3}} \delta_{b_{2}}^{a_{1}} \delta_{b_{3}}^{a_{2}}\right),
$$

which can connect with a chiral vertex. Other vertices which can appear involve the 
gauge fields. The ones relevant for three-loop calculations are

$$
V_{\bar{\phi}_{j} V \phi^{i}}=V_{V^{2} \bar{\phi}_{i} \phi^{j}}=g_{V_{\mathrm{YM}}} \delta_{i}^{j}\left(\delta_{b_{1}}^{a_{2}} \delta_{b_{2}}^{a_{3}} \delta_{b_{3}}^{a_{1}}-\delta_{b_{1}}^{a_{3}} \delta_{b_{2}}^{a_{1}} \delta_{b_{3}}^{a_{2}}\right)
$$

Note that all of the above vertices come with an integral over superspace $\int d^{4} \theta$.

Finally, the composite operator itself can be thought of as a chiral vertex. If it is a scalar composite with $L$ scalar chiral fields, then we denote it as a chiral vertex with $L$ $\operatorname{legs}$,

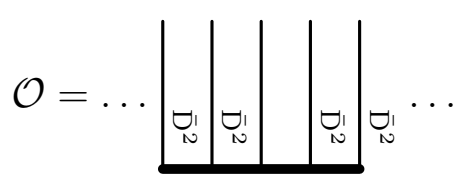

Like the three-point chiral vertex, all but one leg coming out of the composite operator has a $\overline{\mathrm{D}}^{2}$ attached to the leg. Integrating by parts, it is possible to choose any of the legs emerging from the operator to be the one without the $\overline{\mathrm{D}}^{2}$ factor. If we were to consider the more general case of composites that also contain $W_{\alpha}$ fields, then the composite vertex would like

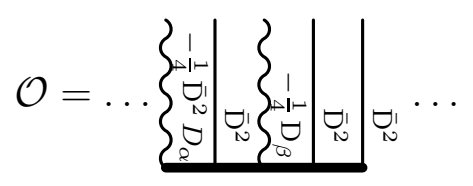

Again, one leg is missing a $\bar{D}^{2}$, in this case it is a $W_{\alpha}$ leg.

As is well known, the dimension of $\mathcal{O}$ has loop corrections, and these loops lead to mixing among the operators. It is hence best to consider the dilatation operator $\mathcal{D}$ that acts on the composite operator and whose eigenvalues are the dimensions of linear combinations of composite operators. $\mathcal{D}$ has a perturbative expansion in $g_{\mathrm{YM}}^{2}$, but in the large $N$ limit, one only need consider planar contributions to $\mathcal{D}$. Hence, the dilation operator can be written as

$$
\mathcal{D}=\sum_{n=0}^{\infty} g^{2 n} \mathcal{D}_{n}
$$


where $g^{2} \equiv \frac{g_{\mathrm{YM}}^{2} N}{16 \pi^{2}}=\frac{\lambda}{16 \pi^{2}}$. Focusing on the case of scalar composites, the lowest order contribution is just $\mathcal{D}_{0}=L$. The first order contribution comes from the supergraph

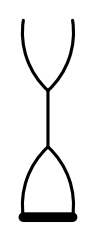

which is a one-loop supergraph with a chiral and an anti-chiral vertex.

The evaluation of any supergraph requires the $D$-algebra $[19,23,28]$, which combines integration by parts with identities for the super-derivatives. Applying this to the supergraph in (2.9), we can choose to have one of the propagators in the loop with a $D^{2}$ and $\overline{\mathrm{D}}^{2}$ factor and the other propagator with no super-derivatives. The $D^{2}$ and $\overline{\mathrm{D}}^{2}$ cancel one of the $\delta^{4}(\theta)$ terms from the propagators while the other $\delta^{4}(\theta)$ term is absorbed by the integral over superspace from the vertex, leaving an ordinary scalar integral. The supergraph will also come with a factor of $G_{i j k} G^{k l m}$, where $i j$ are the two incoming indices of the scalar fields (going left to right) and $l m$ are the outgoing indices (going right to left).

Before stating the result of this integration, let us do a simple power counting exercise for the supergraph. The loop has one vertex (hence one integration over $\int d^{4} \theta$ ), two propagators , 4 super-derivatives and one loop momentum integral. Hence, the dimension is $2-8+2+4=0$. Thus we expect this integral to be logarithmically divergent and lead to a contribution to the anomalous dimension. In fact it does. The divergent part of the scalar integral is $\frac{1}{32 \pi^{2} \epsilon} G_{i j k} G^{k l m}$ where we have dimensionally reduced to $D=4-2 \epsilon$ [28]. If we consider the contribution in the $S U(2)$ sector for $\mathcal{N}=4 \mathrm{SYM}$, where the scalar fields are restricted to $X$ and $Y$, then the dilatation operator becomes

$$
\mathcal{D}_{1}=2 \chi(1)
$$

where $\chi(1)$ is an example of a chiral function. The chiral functions are defined as $[23,28]^{2}$

$$
\chi\left(a_{1}, a_{2} \ldots a_{n}\right) \equiv \sum_{j=0}^{L-1}\left(1-P_{j+a_{1}, j+a_{1}+1}\right)\left(1-P_{j+a_{2}, j+a_{2}+1}\right) \ldots\left(1-P_{j+a_{n}, j+a_{n}+1}\right),
$$

where $P_{j, j+1}$ is the exchange operator between neighboring sites. A useful property of chiral functions is

$$
\chi\left(a_{1}, \ldots a_{j}, a_{j+1} \ldots a_{n}\right)=\chi\left(a_{1}, \ldots a_{j+1}, a_{j} \ldots a_{n}\right)
$$

if $\left|a_{j}-a_{j+1}\right| \geq 2$, while

$$
\chi\left(a_{1}, \ldots a_{j}, a_{j+1} \ldots a_{n}\right)=2 \chi\left(a_{1}, \ldots a_{j} \ldots a_{n}\right)
$$

if $a_{j}=a_{j+1}$. We will call a chiral function connected if it can be arranged using (2.13) to a chiral function where $\left|a_{j}-a_{j+1}\right|=1$ for all $j$. Otherwise, the chiral function is disconnected.

\footnotetext{
${ }^{2}$ Note that our convention differs by a factor of $(-1)^{n}$.
} 
Power counting can be used to exclude many supergraphs $[23,28]$. One of the most powerful rules is that in order to have a divergent supergraph, there must be an antichiral vertex that sits outside of all loops [28]. Clearly the supergraph in (2.9) satisfies this criterion. But then it must also follow that if a supergraph has its composite operator legs connected by gauge propagators only, then this supergraph cannot contribute to $\mathcal{D}$ [28]. For example, the supergraph

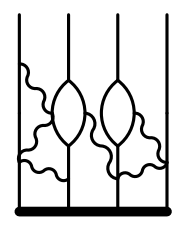

only has connections between neighboring legs via gauge propagators, hence power counting will indicate that this supergraph is finite. The diagram could have subdivergences, but these will cancel with with the subdivergences coming from other diagrams because of the superconformal symmetry. If all legs in a composite are unconnected to their neighboring legs then power counting does not rule out divergences. However, the contribution of each leg comes strictly from the chiral field self-energy, whose divergences cancel by superconformal invariance. Notice that these arguments do not actually rely on the $\mathcal{N}=4$ supersymmetry. As long as one has an $\mathcal{N}=1$ superconformal gauge theory with a well-defined perturbative expansion they are still valid.

\section{A conjecture for chiral supergraphs in $\mathcal{N}=4 \mathrm{SYM}$}

In this section we present a conjecture for a class of supergraphs in $\mathcal{N}=4 \mathrm{SYM}$. In the next section these results will be applied to the cubic Leigh-Strassler model.

To this end, let us consider an operator of length $L$ in the $S U(2)$ sector of $\mathcal{N}=4$ SYM, where $L>>1$ so that wrapping effects can be ignored. Let us assume that we have one magnon on top of the chiral primary groundstate with momentum $p$. Strictly speaking this will not be a physical operator, but we can assume that there is another magnon on the spin-chain with opposite momentum, and if we further assume that the magnon is far away then it has a minimal effect on the other magnon's energy. The magnon can be represented by the state

$$
|p\rangle \equiv \sum_{j=1}^{L} e^{i p j}\left|X X X \ldots X Y \begin{array}{c}
\substack{\downarrow \\
\downarrow} \\
Y
\end{array} \ldots X X X\right\rangle,
$$

which has an energy

$$
\varepsilon(p)=\sqrt{1+16 g^{2} \sin ^{2} \frac{p}{2}}-1 .
$$

We next observe that we can write the identity

$$
\begin{aligned}
\varepsilon(p)|p\rangle & =\left(\sqrt{1+4 g^{2} \chi(1)}-1\right)|p\rangle \\
& =\sum_{m=1}^{\infty} \frac{\Gamma(3 / 2)}{\Gamma(m+1) \Gamma(3 / 2-m)}(2 g)^{2 m}\left[\chi(1)^{m}\right]_{c}|p\rangle
\end{aligned}
$$


where the expression $\left[\chi(1)^{m}\right]_{c}$ refers to the connected part of the product. The unconnected chiral functions give zero when acting on $|p\rangle$. The first several connected products are

$$
\begin{aligned}
{\left[\chi(1)^{2}\right]_{c}=} & 2 \chi(1)+\chi(1,2)+\chi(2,1) \\
{\left[\chi(1)^{3}\right]_{c}=} & 4 \chi(1)+4 \chi(1,2)+4 \chi(2,1)+\chi(1,2,3)+\chi(3,2,1)+\chi(1,2,1)+\chi(2,1,2) \\
{\left[\chi(1)^{4}\right]_{c}=} & 8 \chi(1)+12 \chi(1,2)+12 \chi(2,1)+6 \chi(1,2,3)+6 \chi(3,2,1) \\
& +6 \chi(1,2,1)+6 \chi(2,1,2) \\
& +\chi(1,2,3,4)+\chi(4,3,2,1)+\chi(1,2,3,2)+\chi(2,3,2,1) \\
& +\chi(2,1,2,3)+\chi(3,2,1,2)+\chi(1,2,1,2)+\chi(2,1,2,1)
\end{aligned}
$$

We can also see the expansion diagrammatically using the basic $\chi(1)$ building block, $\mathcal{Y}$. For example, we have

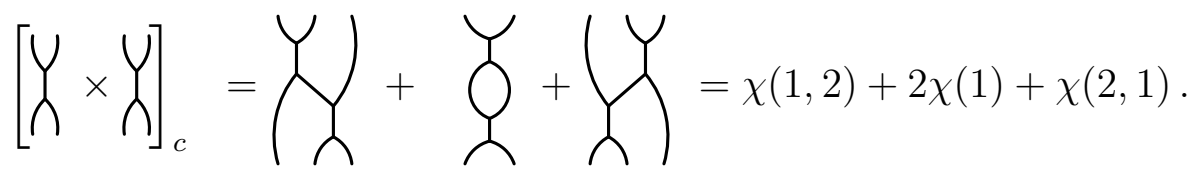

After expanding $\left[\chi(1)^{n}\right]_{c}$ as in (3.4), the expression in (3.3) can be resummed, where we find

$$
\begin{aligned}
& \sum_{m=1}^{\infty} \frac{\Gamma(3 / 2)}{\Gamma(m+1) \Gamma(3 / 2-m)} g^{2 m}\left[\chi(1)^{m}\right]_{c} \\
& =F_{1}\left(g^{2}\right) \chi(1)+F_{2}\left(g^{2}\right)[\chi(1,2)+\chi(2,1)] \\
& +F_{3}\left(g^{2}\right)[\chi(1,2,3)+\chi(3,2,1)+\chi(1,2,1)+\chi(2,1,2)]+\ldots
\end{aligned}
$$

with $F_{n}\left(g^{2}\right)$ given by

$$
\begin{aligned}
F_{n}\left(g^{2}\right) & =\sum_{m=n}^{\infty} \frac{\Gamma(3 / 2)}{m \Gamma(3 / 2-m) \Gamma(n) \Gamma(m-n+1)}(2 g)^{2 m} \\
& =\frac{(-1)^{n+1} \Gamma(n-1 / 2)}{2 \sqrt{\pi} \Gamma(n+1)}(2 g)_{2}^{2 n} F_{1}\left(n-1 / 2, n ; n+1 ;-8 g^{2}\right) .
\end{aligned}
$$

Note that the function $F_{n}\left(g^{2}\right)$ that appears in front of a connected chiral function $\chi\left(a_{1}, \ldots a_{n}\right)$ depends only on $n$ and not the particular values of the $a_{j}$ (although we assume that $\left.\left|a_{j}-a_{j+1}\right|=1\right)$. Even though the function is written in terms of a hypergeometric function for general $n$, all functions are algebraic. The first three examples of these functions are

$$
\begin{aligned}
& F_{1}\left(g^{2}\right)=\frac{1}{2}\left(\sqrt{1+8 g^{2}}-1\right) \\
& F_{2}\left(g^{2}\right)=-\frac{1}{8} \frac{\left(\sqrt{1+8 g^{2}}-1\right)^{2}}{\sqrt{1+8 g^{2}}} \\
& F_{3}\left(g^{2}\right)=\frac{\left(\sqrt{1+8 g^{2}}-1\right)^{3}\left(3 \sqrt{1+8 g^{2}}+1\right)}{64\left(1+8 g^{2}\right)^{3 / 2}} .
\end{aligned}
$$


There is one caveat concerning this construction. Many of the chiral functions are equivalent in the $S U(2)$ sector. In particular, in the $\mathrm{SU}(2)$ sector the chiral functions satisfy the following identity

$$
\chi\left(a_{1} \ldots a_{j}, a_{j}+1, a_{j} \ldots a_{n}\right)=\chi\left(a_{1} \ldots a_{j}, a_{j}-1, a_{j} \ldots a_{n}\right)=\chi\left(a_{1} \ldots a_{j} \ldots a_{n}\right) .
$$

Since a single magnon can always be placed in the $S U(2)$ sector under a global transformation of the $S U(2 \mid 2) \ltimes S U(2 \mid 2)$ symmetry group, it might not be meaningful to break up the energy as in (3.3).

Recently, however, Sieg explicitly computed the $S U(2)$ sector dilatation operator in superspace to three loop order [28]. The three loop prediction was first made in [34] based on integrability, where in the language of chiral functions it is given by ${ }^{3}$

$$
\mathcal{D}_{3}=4(\chi(1,2,3)+\chi(3,2,1))-4 \chi(1,3)+16(\chi(1,2)+\chi(2,1))+24 \chi(1) .
$$

One can see that this is consistent with the dispersion relation in (3.3) by using $\chi(1,2,1)=$ $\chi(2,1,2)=\chi(1)$. In Sieg's computation, he found that the following diagrams contribute to $\mathcal{D}_{3}$ with a $\chi(1)$ factor:

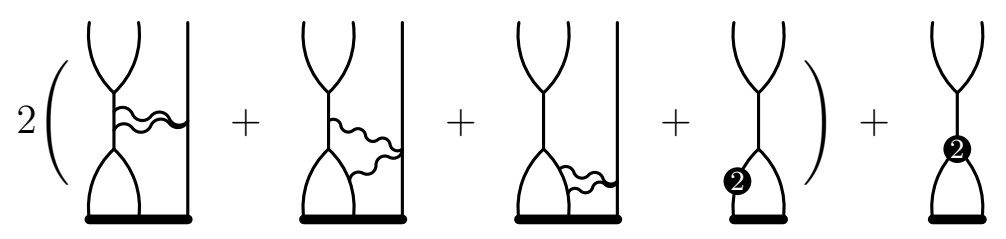

where the circled 2 indicates a two-loop self-energy or vertex correction. Both the selfenergy and the vertex correction must be finite by the superconformal invariance. The overall factor of 2 in front of the first four diagrams takes into account their reflections. Note that all of these supergraphs have two neighboring legs connected by a chiral vertex, but if there is a third leg it is only connected by vector propagators. The resulting contribution to $\mathcal{D}_{3}$ from these supergraphs is [28]

$$
\Delta^{(1)} \mathcal{D}_{3}=16 \chi(1)
$$

There are two further supergraphs that contribute to $\chi(1)$, which do so through a $\chi(1,2,1)$ or a $\chi(2,1,2)$ structure. These are

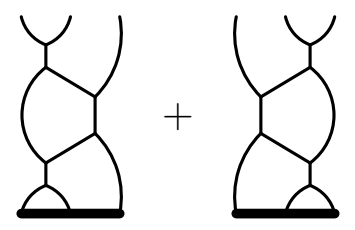

which give the contribution [28]

$$
\Delta^{(1,2,1)} \mathcal{D}_{3}+\Delta^{(2,1,2)} \mathcal{D}_{3}=4 \chi(1,2,1)+4 \chi(2,1,2) .
$$

Hence, even though $\chi(1,2,1)$ and $\chi(2,1,2)$ are identified with $\chi(1)$ in the $S U(2)$ sector, the linear combination of connected chiral functions from the three loop dilatation operator is the same linear combination as in $\left[\chi(1)^{3}\right]_{c}$.

\footnotetext{
${ }^{3}$ We drop a term that does not affect the spectrum.
} 
Base on this evidence, $\mathrm{t}$ is tempting to conjecture that the all-loop sum of supergraphs with the connected chiral function $\chi\left(a_{1}, a_{2} \ldots a_{j}, \ldots a_{n}\right)$ is given by

$$
F_{n}\left(g^{2}\right) \chi\left(a_{1}, a_{2} \ldots a_{j}, \ldots a_{n}\right)
$$

where $F_{n}\left(g^{2}\right)$ is defined in (3.7). Note that the sum would include the contributions of all possible insertions of gauge vertices and propagators as well as chiral loops. However, studies at four loops and higher show that this is not the case [32]. Instead we make the weaker statement that (3.15) captures the rational contribution to the chiral functions. Starting at four loops there are higher transcendental terms that contribute to the chiral functions, but cancel out in the $\mathcal{N}=4$ dispersion relation because of the identity in (3.9). We can still use the $\mathcal{N}=4$ dispersion and the weaker conjecture to make statements about higher loop contributions to the anomalous dimensions in $\mathcal{N}=1$ models.

\section{Application to the cubic Leigh-Strassler model}

\subsection{The cubic model and its corrections to the coupling}

In this section we apply the results from the previous two sections to the cubic LeighStrassler model.

Let us quickly review where the identification $\widehat{h}=\sqrt{2} g_{\text {YM }}$ comes from. In order for the theory to be superconformal, the anomalous dimensions of the chiral fields must be zero. In superspace, this means that the self-energy for the chiral fields is finite. If we had chosen the superpotential in (1.1) to have $h=0, q=1$ and $\kappa=g_{\mathrm{YM}}$, then this would be the superpotential for $\mathcal{N}=4 \mathrm{SYM}$ where we know that all self-energies are finite. Hence, it is just a question of comparing the difference using the different chiral vertices. At the one-loop level the relevant supergraph for the self-energy of, say, a $Z$ chiral field is the skeleton ${ }^{4}$

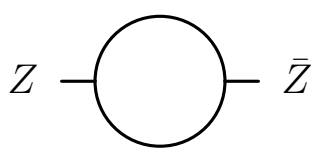

In the case of planar $\mathcal{N}=4 \mathrm{SYM}$ there are two ways to assign the $X$ and $Y$ flavors on the internal propagators, but for the superpotential in (1.4) there is only one way. Hence, to compensate for this difference, $\widehat{h}$ should be chosen equal $\sqrt{2} g_{\mathrm{YM}}$ so that the contributions of this supergraph to the self-energy are the same. The divergent part from this supergraph will then cancel with the one-loop supergraphs having only gauge vertices.

Remarkably, this counting works all the way up to the four loop-level, where one encounters a new type of graph that requires a modification of the $\widehat{h}$ value of order $\Delta \widehat{h} \sim g_{\mathrm{YM}}^{7} N^{3}[18]$. At four loops one has the self-energy skeleton [13,14]

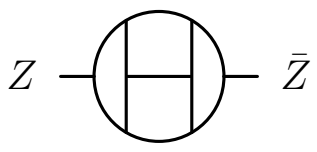

\footnotetext{
${ }^{4}$ We will call any supergraph with only chiral propagators a skeleton.
} 
which has four chiral vertices and 4 anti-chiral vertices. In the case of planar $\mathcal{N}=4$ SYM one finds that there are 8 ways to assign the flavors on the internal propagators. However, since $\widehat{h}$ has been set to $\sqrt{2} g_{Y M}$, this supergraph will come with a factor of 16 for the cubic model. Hence, there is no longer a matching. To compensate, one must adjust $\widehat{h}$ to $\sqrt{2} g_{\text {YM }}+\Delta \widehat{h}$ so that the scalar field anomalous dimension remains zero to order $g^{8}$. Diagrammatically the correction looks like

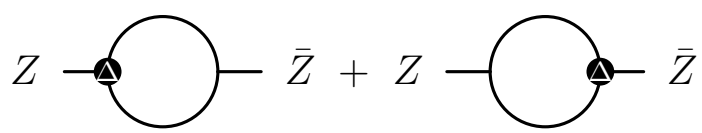

where the blob with the $\Delta$ denotes the tree-level correction to the chiral vertex coming from the correction to $\widehat{h}$.

As was pointed out in $[13,14]$, canceling the contributions to the anomalous dimension is not the same as canceling the divergence in the chiral propagator since the contributions come at different loop orders. Using dimensional reduction, the divergence coming from the graphs in (4.3) and the extra contribution in (4.2) equals

$$
\frac{A g^{2} \delta \widehat{h}}{g_{\mathrm{YM}} \epsilon}\left(\frac{\mu^{2}}{p^{2}}\right)^{\epsilon}+\frac{B g^{8}}{\epsilon}\left(\frac{\mu^{2}}{p^{2}}\right)^{4 \epsilon},
$$

where $A$ and $B$ are some constants, whose actual values can be found in [18], but are not necessary for us here. Hence, to cancel the anomalous dimension, one should set

$$
A \delta \widehat{h}+4 g_{\mathrm{YM}} g^{6} B=0 .
$$

This leaves the divergence in (4.4) uncanceled.

There are two proposed ways to deal with this mismatch of divergences, which turn out to be equivalent, at least for everything computed in the latter part of this section. One way is to include $\epsilon$ dependent corrections in $\widehat{h}$ with lower powers of $g_{\text {YM }}$ [18,35-37]. In this case the $\epsilon^{-1}$ poles can be canceled with $\epsilon^{-4}$ poles and lower. In this sense the theory is finite.

The other option is to include infinite counterterms for the chiral propagators and gauge vertices [14], which are related by the Slavnov-Taylor identities. We will use this procedure here because the bookkeeping strikes us as simpler. Hence, at the four loop level we would add the counterterm $-\left(A \widehat{h}+B g^{6}\right) \epsilon^{-1}$ to the chiral propagator to cancel the divergence. At five-loops not only will there be new single poles, but there will be double poles as well that need to be canceled. One can keep going to higher and higher powers of $g^{2}$ in a systematic fashion, canceling the anomalous dimension and the poles, as well as contributions coming from more complicated skeletons, by adding corrections to $\widehat{h}$ and counterterms. This process does introduce scheme dependence [14] because the counterterms can include finite pieces which affect the next order anomalous dimension cancelation. Hence the five-loop correction to $\widehat{h}$ is scheme dependent. However, in the five-loop computation we present later the result is scheme independent, assuming that one uses the same regularization scheme for the operators as for the chiral fields. 


\subsection{Planar protected states}

In the scalar sector, the one-loop Hamiltonian of the cubic model is that of an $S U(3)$ generalization of the anti-ferromagnetic Ising model. Its explicit form is

$$
g^{2} \mathcal{D}_{1}=4 g^{2} \sum_{j=1}^{L}\left(\frac{1}{3}+2 \tau_{j}^{3} \tau_{j+1}^{3}+\frac{3}{2} \tau_{j}^{8} \tau_{j+1}^{8}\right) \equiv 2 g^{2} \chi(1),
$$

where $\tau^{3}$ and $\tau^{8}$ are the $S U(3)$ generators

$$
\tau^{3}=\operatorname{diag}\left(\frac{1}{2},-\frac{1}{2}, 0\right), \quad \tau^{8}=\operatorname{diag}\left(\frac{1}{3}, \frac{1}{3},-\frac{2}{3}\right) .
$$

This defines the chiral function $\chi(1)$ for the cubic model. The spin-chain has no kinetic term and one can easily verify that the contribution from neighboring sites with the same flavors is $4 g^{2}$, while the contribution from neighboring sites with different flavors is 0 .

In order to investigate the higher loops, we can borrow many of the arguments from the preceding sections since the coupling in the cubic model is assumed to be tuned so that there is $\mathcal{N}=1$ superconformal symmetry. For example, if no nearest neighbors in a supergraph are connected by a chiral vertex, then the supergraph is finite. The corrections to $\widehat{h}$ and the counterterms do not change this argument because the subdivergences will continue to cancel. Given the superpotential in (1.4), this implies that if all nearest neighbors in the scalar composite have different flavors then all supergraphs are finite at the planar level since there is no way to connect neighboring fields with a chiral vertex. Phrased differently, all chiral functions for the cubic model acting on such an operator are zero. Hence, in the planar limit, all such operators have zero anomalous dimension for all values of the coupling.

We will refer to these operators as "planar protected". They are not chiral primaries, because nonplanar supergraphs can contribute to their anomalous dimensions. This is also evident from the symmetry, which does not have individual conserved charges for the different flavors. For scalar composites of length $L, L>>1$, there are $\sim 2^{L} / L$ different planar protected composites. While perhaps obvious to many, we explain the counting in the appendix.

\subsection{The one-pair states}

We next consider scalar composites where one pair of nearest neighbors have the same flavor but all other nearest neighbors have different flavors. We call these one-pair states. The only possibility for a divergent supergraph is that the nearest neighbors in the pair are connected by a chiral vertex. Since the outgoing fields are the same as the incoming ones, these composites do not mix at the planar level to all orders in the coupling. Hence, the position of the pair is fixed on the composite, up to the overall cyclic symmetry.

For these states, only the $\chi(1)$ supergraphs contribute. Hence, up to three loop level we can borrow the $\chi(1)$ contribution of the $\mathcal{N}=4$ dilatation operator, replacing the $\chi(1)$ with the one found in (4.6) to compute the anomalous dimension of this operator. 
Taking the expansion of $F_{1}\left(g^{2}\right)$ in (3.7) and using that $\chi(1)=2$ for the one-pair states gives

$$
\delta_{X X}^{(3)}=4 g^{2}-8 g^{4}+16 g^{6}+\mathrm{O}\left(g^{8}\right)
$$

where the subscript indicates that this is for a one-pair state. We can use the $\mathcal{N}=4$ dispersion relation to find the four-loop contribution. If we use the conjecture from the previous section we can also find the rational contributions to $\delta_{X X}$ beyond four loops.

However, since there is a four-loop correction to $\widehat{h}$ it is first necessary to adjust the contributing supergraphs. At four loops one has the supergraph

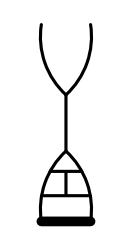

One can easily check that this supergraph has an extra factor of 2 in the cubic model as compared to the $\mathcal{N}=4$ case, just like the self-energy. To this we should add the correction

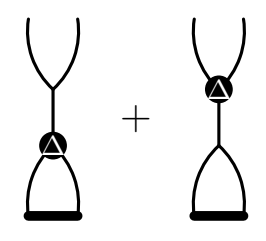

Since the topologies of the loops and comparative factors in (4.9) and (4.10) are the same as in (4.2) and (4.3) respectively, the divergent part in (4.10) is exactly the same as in (4.4). Hence, canceling the anomalous dimension for the chiral fields ensures that there is no correction to the anomalous dimension at order $g^{8}$. All other four-loop supergraphs lead to the same result as in $\mathcal{N}=4 \mathrm{SYM}$.

There is still an uncanceled divergence which should be removed by adding a counterterm for the operator. To find the appropriate counterterm, we note that the equations of motion set

$$
X X=\frac{i}{\hat{h}} \int d^{2} \bar{\theta} e^{-g_{\mathrm{YM}} V} \bar{X} e^{g_{\mathrm{YM}} V} .
$$

Since the righthand side comes from the $D$ term in the Lagrangian, it will have the same wave-function renormalization factor as the chiral propagator. Hence, the operator counterterm can be implemented by replacing $X X$ with the righthand side of (4.11) and with the same counterterm factor as for the chiral propagator. Diagramatically, we draw this term as

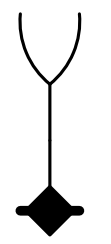

where one should keep in mind that the upper vertex does not come with a factor of $\widehat{h}$ since it has been divided out by the $\widehat{h}$ in the denominator of the counterterm. The 
divergence in (4.12) along with those in (4.10) now cancel in a way parallel to the cancellation of divergences for the chiral propagator.

We can extend the argument to five loops. In this case there can be one vector propagator added to (4.9) and (4.10) in various configurations. For example, one has the supergraphs

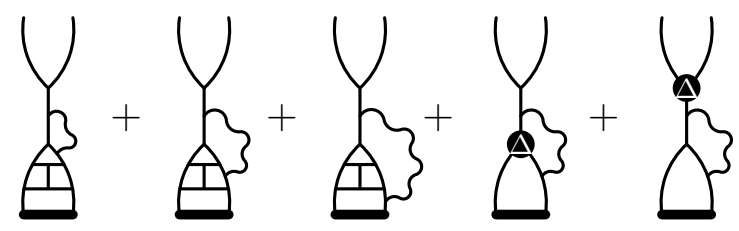

as well as their reflections. These supergraphs have the same topologies as the following contributions to the 5 loop self-energy

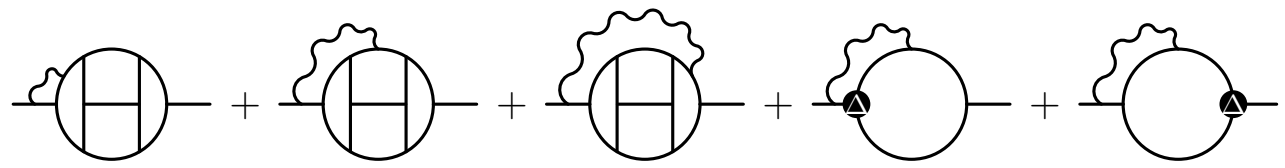

where the corresponding diagrams for the reflections have the vector propagators below the external legs. There are many other supergraphs similar to (4.13), but where both ends of the vector propagator are attached to points below the anti-chiral vertex. Each one of these supergraphs is equivalent to a particular five-loop self-energy diagram. Moreover, there is an equivalence for the terms involving counterterms, namely

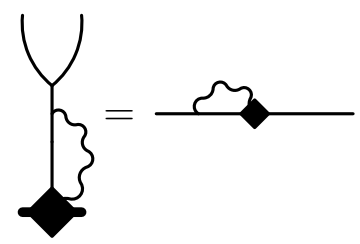

Then there are the five-loop supergraphs involving three legs, for example

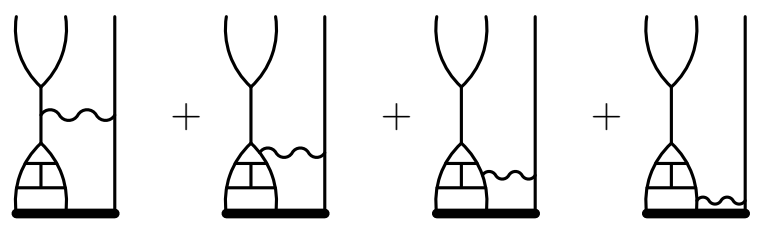

as well as their reflections. Using the $D$-algebra, one can show that the first two and the last two each sum to a UV finite result [23,28], although this is not crucial for our argument. These supergraphs are topologically equivalent to the five-loop self-energy supergraphs

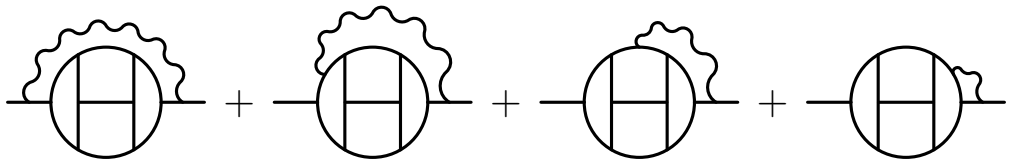

If we include the reflections of the supergraphs in (4.17), then the divergence from all possible five-loop supergraphs involving the basic four-loop skeleton in (4.9) equals the divergence from all possible self-energy supergraphs built from the four-loop self-energy 
skeleton in (4.2). The same holds true for the two-loop supergraphs built out of (4.10) and the one-loop term built from the counterterm in (4.12). Finally, both the chiral propagator and the operator require the same double-pole counterterm to cancel this divergence. Hence, if $\widehat{h}$ and the counterterms are tuned to cancel the divergences in the five-loop self-energy, then they also cancel the unwanted divergences of the operator supergraphs. We emphasize that this is independent of the regularization scheme one chooses.

This matching of operator supergraphs to self-energy supergraphs can be applied to higher loops as well, as long as the supergraphs involve only two or three legs of the scalar composite. However, starting at six loops one can have supergraphs involving four legs. The contribution from those built from the skeleton in (4.9) are

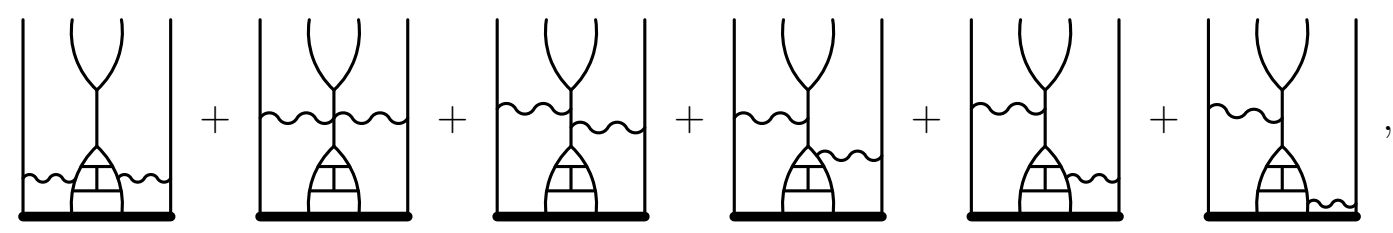

plus the reflections of the last four. One can show using the $D$-algebra that the divergences in these supergraphs cancel in pairs [23,28]. Furthermore, using the $D$-algebra the last four can be shown equivalent to the six-loop self-energy diagrams

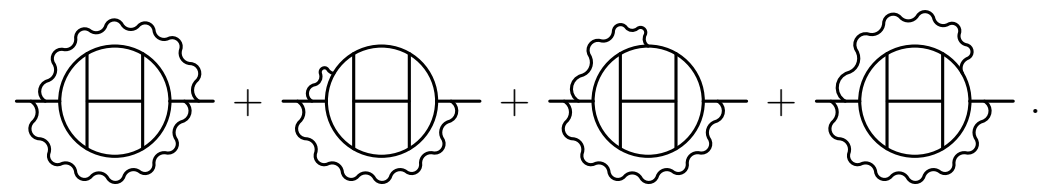

The first two diagrams in (4.18) do not have an obvious mapping to self-energy diagrams, although this is not important since their UV divergent parts cancel. Likewise, there are six-loop self-energy diagrams that do not have obvious partners for operator diagrams. At this time we have not established whether or not the UV divergences for these unmatched self-energy diagrams cancel because of $D$-algebra identities.

Having established the cancellation of these extra terms to at least five-loop order, we now claim using the conjecture from the previous section that the anomalous dimension for the one-pair class of operators is

$$
\delta_{X X}^{(5)}=2 F_{1}\left(g^{2}\right)+T^{(1)}\left(g^{2}\right)+\mathrm{O}\left(g^{12}\right)=\sqrt{1+8 g^{2}}-1+T^{(1)}\left(g^{2}\right)+\mathrm{O}\left(g^{12}\right) .
$$

The function $T^{(1)}\left(g^{2}\right)$ contains higher transcendental terms starting at four-loop order.

We can find the four-loop contribution to $T^{(1)}\left(g^{2}\right)$ indirectly. The connected chiral functions that receive four-loop contributions include $\chi(1), \chi(1,2,1)$ and $\chi(2,1,2)$. By symmetry, $\chi(1,2,1)$ and $\chi(2,1,2)$ have the same four-loop contribution and both are equivalent to $\chi(1)$ in the $S U(2)$ sector. If $\chi(1,2,1)$ has a transcendental contribution, since no such term appears in the $\mathcal{N}=4$ dispersion, it must cancel out with a contribution from $T^{(1)}\left(g^{2}\right)$. In [32] we show that the four-loop transcendental contribution from $\chi(1,2,1)$ is $8 \zeta(3) g^{8}$, hence to cancel this, we must have

$$
T^{(1)}\left(g^{2}\right)=-16 \zeta(3) g^{8}+\mathrm{O}\left(g^{10}\right) .
$$


Therefore, the anomalous dimension, up to and including four-loop order, is

$$
\delta_{X X}^{(4)}=4 g^{2}-8 g^{4}+16 g^{6}-16(5+\zeta(3)) g^{8}+\mathrm{O}\left(g^{10}\right),
$$

Note that since the four-loop transcendental contribution is negative, we expect that the anomalous dimension scales less than $g$ for large $g$.

Note that result in (4.20) will be modified by wrapping corrections [22, 23, 38-40]. Just like the case of $\mathcal{N}=4 \mathrm{SYM}$, there is a correction at $L$ loop order for a composite operator of length $L$.

\subsection{Multi-paired states}

We can also consider multiple pairs of fields. The positions of the pairs are still fixed. If pairs are neighbors then they must be composed of different scalar fields. Even though the pairs are fixed they still have a separation dependent potential. At weak coupling the potential scales as $g^{2(2+\ell)}$, where $\ell$ is the separation. This means that the interaction first shows up at three-loop order. The three-loop dilatation operator in (3.10) contains the term

$$
\mathcal{D}_{3}^{(1,3)}=-4 g^{6} \chi(1,3) .
$$

Hence, the change in energy for each neighboring pair is $-16 g^{6}$ to lowest nonzero order. We conclude that for any scalar composite operator with no more than two sequential flavors of the same type anywhere in the trace, the anomalous dimension is known to at least three-loop order. This means that for scalar composites of length $L$, we know the anomalous dimensions for approximately $\frac{1}{L}\left(\frac{11}{4}\right)^{L}$ different operators, along with their complex conjugates (see the appendix for an explanation of the counting).

\subsection{Quadruplets}

When there are three or more sequential scalar fields with the same flavor, the composite operator can mix with other composites and the spin-chain becomes dynamic. For instance, $X X X$ can mix into $\epsilon^{\alpha \beta} W_{\alpha} W_{\beta}$ through the supergraph

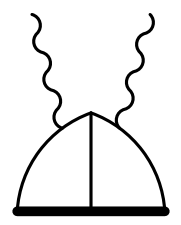

where a $\bar{D}^{2} D_{\alpha}$ and a $D_{\beta}$ are pulled out of the loop and onto the vector legs. This assumes that one of the three scalar fields is missing a $\bar{D}^{2}$, otherwise we would pull out an extra $\bar{D}^{2}$ onto the second vector.

Once produced, the $W_{\alpha}$ 's can migrate along the chain, or the pair can mix back into $X X X, Y Y Y$ or $Z Z Z$. To see what happens in this latter case, suppose we start with a composite operator that alternates between $X$ and $Y$, except for one sequence of $Y Y Y Y$, 
Then either the left three $Y^{\prime}$ 's or the right three can transform into an $X X X$ through a $W^{2}$, which is essentially a two-loop effect [33]. This leaves an $X X X X$ either to the left or the right of the original $Y Y Y Y$. The process can then continue. For example, we could have the pattern

$$
\begin{gathered}
\ldots X Y X Y X[Y Y Y Y] X Y X Y X Y \ldots \\
\downarrow \\
\ldots W^{2} \\
\ldots X Y X Y[X X X X] Y X Y X Y X Y \ldots \\
\downarrow W^{2} \\
\ldots X Y X[Y Y Y Y] X Y X Y X Y X Y \ldots \\
\downarrow \\
\downarrow W^{2} \\
\ldots X Y X Y[X X X X] Y X Y X Y X Y \ldots
\end{gathered}
$$

where we see that the quadruplet of scalar fields can propagate in either direction along the chain. Its position determines whethere the quadruplet is $X X X X$ or $Y Y Y Y$. While $Y Y Y$ and $X X X$ can also mix into $Z Z Z$, the $Z Z Z$ will be fixed in place and will eventually transform back into $X X X$ or $Y Y Y$. Since the triplet $Z Z Z$ has less energy than the quadruplet at the one-loop level, where the scalar composite is nondynamical, this will be a higher order effect.

If we had started with a scalar composite with only the triplet $Y Y Y$, then it could behave as follows

$$
\begin{aligned}
& \ldots X Y X Y X[Y Y Y] X Y X Y X Y \ldots \\
& \downarrow W^{2} \\
& \ldots X Y X Y[X X X X X] Y X Y X Y \ldots \\
& \downarrow W^{2} \\
& \ldots X Y X[Y Y Y Y][X X] Y X Y X Y \ldots \\
& \downarrow W^{2} \\
& \ldots X Y[X X X X] Y[X X] Y X Y X Y \ldots
\end{aligned}
$$

Here we see that the triplet has split into a pair and a quadruplet. The pair stays fixed while the quadruplet migrates along the chain. The pair then acts as a fixed impurity which the quadruplet can scatter through.

One can continue to add quadruplets and pairs onto the chain. It should be straightforward to derive the Hamiltonian for this system up to two-loop order using the dilation operator of the $S U(2 \mid 3)$ sector in [33], although we have not done this here.

\subsection{A finite Hagedorn temperature at strong coupling?}

The exponential growth in $L$ of the planar protected states would indicate that the gravity dual of this theory is very unusual. The gravity dual for the superpotential in (1.1) with $q=1$ and $|h|<<1$ has been investigated in [41,42]. For the dual one turns on fluxes and deforms the $S^{5}$. The cubic model is far away from this regime. In any case, even at large coupling the exponential growth of the planar protected operators would seem to indicate that the theory on $S^{1} \times S^{3}$ has a Hagedorn temperature of order one in units of the $S^{3}$ inverse radius, even at strong coupling. At zero coupling the particle content is the same as $\mathcal{N}=4 \mathrm{SYM}$ and hence would have the same Hagedorn temperature $[43,44]$. Going to infinite coupling in $\mathcal{N}=4$ SYM takes the Hagedorn 
temperature to infinity, since the growth of chiral primaries is polynomial in $L$. Of course this is expected since the dual theory reduces to supergravity. For the cubic model, since there appears to be a finite Hagedorn temperature it would indicate that the low energy gravity dual is stringy in nature.

Even though the cubic model appears to have a Hagedorn temperature, it is far from obvious that there would be a sharp phase transition here. At weak coupling, the Hagedorn temperature indicates a breakdown in planarity. But if planarity is breaking down, then the nonplanar contributions to the anomalous dimensions become important. This could then tamp down the exponential growth in the density of states and push the transition point upward, perhaps all the way to $(\lambda)^{1 / 4}$ in the strong coupling limit.

\section{Discussion}

In this paper we considered the cubic branch of the Leigh-Strassler superconformal theories, arguing that it has an exponentially large number of states with zero anomalous dimension in the planar limit. We then considered states where impurities were added to these zero energy states. Using information about the $\mathcal{N}=4$ magnon dispersion relation we were able to compute the anomalous dimension of these operators to 4-loop order. Furthermore, using a conjecture relating sums of connected graphs we are able to make an all orders prediction for the rational parts of their anomalous dimensions, assuming that unwanted divergences will continue to cancel beyond five-loop order.

There are several directions that one can pursue. Since part of our results depends on the conjecture, it would be very helpful to have a proof (or disproof) of its validity. Further evidence for the conjecture will be presented in [32]. It would also be desirable to have a better understanding of how the transcendental parts enter into the $\mathcal{N}=1$ computations. Our conjecture only uses the dispersion relation and not the $S$-matrix, so perhaps one could find similar relations between the $S$-matrix and classes of supergraphs that are associated with more general chiral functions and not just the connected ones. It would also be useful to find an argument showing that the unwanted divergences continue to cancel beyond five-loop order when adjusting the coupling to cancel the anomalous dimension of the chiral fields.

Another possible direction is to find an analog of the conjecture for the ABJM/ABJ models $[45,46]$. In particular, this could be useful in ascertaining the higher loop corrections to the undetermined function $h^{2}(\lambda, \hat{\lambda})$ that appears in the magnon dispersion relation [24-26] and the $S$-matrix. Inspecting the supergraphs in [26], there is some indication that they are organized in a way related to the dispersion relation and the two 't Hooft couplings. The spin-chains in ABJM/ABJ are of alternating type, which leads to two types of magnons. The dispersion relation is then investigated in the $S U(2) \times S U(2)$ sector. The first relevant equivalence for chiral functions is $\chi(1,3,1)=\chi(3,1,3)=\chi(1)$, and one would have to do a six-loop calculation to find the equivalent data point of the three-loop calculation in $\mathcal{N}=4$. As a very simple check, if the conjecture is true then the contribution from the $\chi(1,3,1)$ and $\chi(3,1,3)$ supergraphs could only be proportional to $\lambda^{3} \hat{\lambda}^{3}$ based on the leading expansion of $h^{2}(\lambda, \hat{\lambda})$. One can quickly verify by drawing the relevant diagrams with the $\chi(1,3,1)$ and $\chi(3,1,3)$ chiral functions that this is the case. 
The ABJM/ABJ model is complicated by the fact that the chiral vertices are fourpoint and so the supergraphs do not have the multiplication structure of the fundamental building blocks as in (3.5). A model that is similar to ABJM/ABJ in that it also has two couplings is the interpolating $\mathcal{N}=2$ superconformal $S U(N) \times S U(N)$ quiver theory discussed in [47,48]. When the couplings are equal then this is the $Z_{2}$ orbifold of $\mathcal{N}=4$ SYM. As one varies the ratio of couplings it interpolates between this point and $\mathcal{N}=2$ SYM with $2 N$ hypermultiplets. The orbifold point has exactly the same supergraph structure as the unorbifolded theory and one can thus borrow all of the results from there. Moving away from the orbifold point, one starts seeing differences at three-loop level [49]. The chiral and anti-chiral vertices are always trivalent so the chiral structure is the same as $\mathcal{N}=4$ and any differences can be swept into the chiral functions which would now also depend on the ratio of couplings [49]. Hence, one can use the conjecture here, and the problem reduces to finding the chiral functions, although they are are highly nontrivial and are known only to the lowest loop order [49].

It would be very interesting if the cubic model were to have any integrable structures. It is unlikely that the full theory is integrable, but perhaps there is integrability in the closed sector of chiral composite operators. It would also be interesting to find the gravity dual for the cubic model. As stated in the main text, we expect it to have some stringy features, even at low energies. In essence, there can be no separation into a supergravity limit with a finite number of fields.

\section{Acknowledgments}

I thank Christoph Sieg for a careful reading of the manuscript and his many helpful comments. I also thank the CTP at MIT for kind hospitality during the course of this work. This research is supported in part by Vetenskaprådet. 


\section{A Appendix}

Here we explain the counting of states for certain types of scalar composites of length $L$.

To count states where all nearest neighbors have different flavors, start with the first site of the chain. This has 3 choices. All subsequent sites up to $L-1$ have 2 choices, since they must be different than the preceding site. At the last site the flavor must be different than the flavor at the first site and at the $L-1$ site. Since we are assuming that $L>>1$ we can assume that for random sequential choices starting from the first site and moving to the right, the first site and the $L-1$ site are uncorrelated. Hence, there is a $2 / 3$ chance that they have different flavors in which case there is only one choice for the last site, and a 1/3 chance that they have the same flavor, in which case there are 2 choices for the last site. Thus, the average number of choices for the last site is $4 / 3$. Combining the first and the last site we see that on average there are 4 choices for the two of them, leaving $\approx 2^{L}$ different combinations for the whole chain. However, because of the trace, composite operators are identified if all fields in the operator are shifted by one site. This means that for almost all chains of length $L$ (all chains if $L$ is prime), they are identified with $L-1$ other chains. Therefore, we should divide $2^{L}$ by $L$.

To count scalar composites which do not have three sequential sites with the same flavor, we again assume that $L$ is large. At any one site on the chain there are 3 choices for the flavor if the preceding two sites have different flavors, and 2 choices if the preceding two sites have the same flavor. To this end, let $r$ be the probability that the preceding two sites are the same, then the average number of choices for the site are $2 r+3(1-r)=3-r$. In order to be consistent, we must find the same $r$ for the probability that the site and its precedent have the same flavor. They can only have the same flavor if the preceding two are different, in which case there is a $1 / 3$ chance. Therefore, we have the consistency relation $(1-r) / 3=r$. Hence, $r=1 / 4$ and the average number of choices is $11 / 4$. Again we must divide by $L$ because of the trace condition, giving us $\approx(11 / 4)^{L} / L$ different scalar composites of this type.

Note that the total number of scalar composites with no conditions is $\sim 3^{L} / L$.

\section{References}

[1] N. Beisert et. al., Review of AdS/CFT Integrability: an Overview, arXiv:1012.3982.

[2] R. G. Leigh and M. J. Strassler, Exactly Marginal Operators and Duality in Four-Dimensional $\mathcal{N}=1$ Supersymmetric Gauge Theory, Nucl. Phys. B447 (1995) 95-136, [hep-th/9503121].

[3] O. Aharony and S. S. Razamat, Exactly Marginal Deformations of $\mathcal{N}=4$ Sym and of Its Supersymmetric Orbifold Descendants, JHEP 05 (2002) 029, [hep-th/0204045].

[4] K. Zoubos, Review of AdS/CFT Integrability, Chapter Iv.2: Deformations, Orbifolds and Open Boundaries, arXiv:1012.3998.

[5] V. V. Khoze, Amplitudes in the Beta-Deformed Conformal Yang-Mills, JHEP 02 (2006) 040, [hep-th/0512194].

[6] A. Mauri, S. Penati, A. Santambrogio, and D. Zanon, Exact Results in Planar $\mathcal{N}=1$ Superconformal Yang-Mills Theory, JHEP 11 (2005) 024, [hep-th/0507282].

[7] R. Roiban, On Spin Chains and Field Theories, JHEP 09 (2004) 023, [hep-th/0312218]. 
[8] D. Berenstein and S. A. Cherkis, Deformations of $\mathcal{N}=4$ Sym and Integrable Spin Chain Models, Nucl. Phys. B702 (2004) 49-85, [hep-th/0405215].

[9] N. Beisert and R. Roiban, Beauty and the Twist: the Bethe Ansatz for Twisted $\mathcal{N}=4$ Sym, JHEP 08 (2005) 039, [hep-th/0505187].

[10] S. A. Frolov, R. Roiban, and A. A. Tseytlin, Gauge - String Duality for Superconformal Deformations of $\mathcal{N}=4$ Super Yang-Mills Theory, JHEP 07 (2005) 045, [hep-th/0503192].

[11] O. Lunin and J. M. Maldacena, Deforming Field Theories with U(1) × U(1) Global Symmetry and Their Gravity Duals, JHEP 05 (2005) 033, [hep-th/0502086].

[12] S. Frolov, Lax Pair for Strings in Lunin-Maldacena Background, JHEP 05 (2005) 069, [hep-th/0503201].

[13] F. Elmetti, A. Mauri, S. Penati, and A. Santambrogio, Conformal Invariance of the Planar Beta-Deformed $\mathcal{N}=4$ Sym Theory Requires Beta Real, JHEP 01 (2007) 026, [hep-th/0606125].

[14] F. Elmetti, A. Mauri, S. Penati, A. Santambrogio, and D. Zanon, Real Versus Complex Beta-Deformation of the $\mathcal{N}=4$ Planar Super Yang-Mills Theory, JHEP 10 (2007) 102, [arXiv:0705.1483].

[15] D. Bundzik and T. Mansson, The General Leigh-Strassler Deformation and Integrability, JHEP 01 (2006) 116, [hep-th/0512093].

[16] T. Mansson, The Leigh-Strassler Deformation and the Quest for Integrability, JHEP 06 (2007) 010, [hep-th/0703150].

[17] T. Mansson and K. Zoubos, Quantum Symmetries and Marginal Deformations, JHEP 10 (2010) 043, [arXiv:0811.3755].

[18] L. V. Bork, D. I. Kazakov, G. S. Vartanov, and A. V. Zhiboedov, Conformal Invariance in the Leigh-Strassler Deformed $\mathcal{N}=4$ Sym Theory, JHEP 04 (2008) 003, [arXiv:0712.4132].

[19] S. J. Gates, M. T. Grisaru, M. Roček, and W. Siegel, Superspace, Or One Thousand and One Lessons in Supersymmetry, Front. Phys. 58 (1983) 1-548, [hep-th/0108200].

[20] C. Sieg, Review of AdS/CFT Integrability, Chapter I.2: the Spectrum from Perturbative Gauge Theory, arXiv: 1012.3984.

[21] J. A. Minahan and K. Zarembo, The Bethe-Ansatz for $\mathcal{N}=4$ Super Yang-Mills, JHEP 03 (2003) 013, [hep-th/0212208].

[22] F. Fiamberti, A. Santambrogio, C. Sieg, and D. Zanon, Wrapping at Four Loops in $\mathcal{N}=4$ Sym, Phys. Lett. B666 (2008) 100-105, [arXiv:0712.3522].

[23] F. Fiamberti, A. Santambrogio, C. Sieg, and D. Zanon, Anomalous Dimension with Wrapping at Four Loops in $\mathcal{N}=4$ Sym, Nucl. Phys. B805 (2008) 231-266, [arXiv:0806.2095].

[24] J. A. Minahan, O. Ohlsson Sax, and C. Sieg, Magnon Dispersion to Four Loops in the Abjm and Abj Models, J. Phys. A43 (2010) 275402, [arXiv:0908.2463].

[25] J. A. Minahan, O. Ohlsson Sax, and C. Sieg, Anomalous Dimensions at Four Loops in $\mathcal{N}=6$ Superconformal Chern-Simons Theories, Nucl. Phys. B846 (2011) 542-606, [arXiv:0912.3460].

[26] M. Leoni et. al., Superspace Calculation of the Four-Loop Spectrum in $\mathcal{N}=6$ Supersymmetric Chern-Simons Theories, JHEP 12 (2010) 074, [arXiv:1010.1756].

[27] M. Benna, I. Klebanov, T. Klose, and M. Smedback, Superconformal Chern-Simons Theories and $\mathrm{AdS}_{4} / C F T_{3}$ Correspondence, JHEP 09 (2008) 072, [arXiv:0806.1519].

[28] C. Sieg, Superspace Computation of the Three-Loop Dilatation Operator of $\mathcal{N}=4$ Sym Theory, arXiv: 1008.3351.

[29] N. Beisert, V. Dippel, and M. Staudacher, A Novel Long Range Spin Chain and Planar $\mathcal{N}=4$ Super Yang- Mills, JHEP 07 (2004) 075, [hep-th/0405001]. 
[30] F. Fiamberti, A. Santambrogio, C. Sieg, and D. Zanon, Finite-Size Effects in the Superconformal Beta-Deformed $\mathcal{N}=4$ Sym, JHEP 08 (2008) 057, [arXiv:0806.2103].

[31] F. Fiamberti, A. Santambrogio, C. Sieg, and D. Zanon, Single Impurity Operators at Critical Wrapping Order in the Beta-Deformed $\mathcal{N}=4$ Sym, JHEP 08 (2009) 034, [arXiv:0811.4594].

[32] J. A. Minahan and C. Sieg. To appear.

[33] N. Beisert, The SU(2|3) Dynamic Spin Chain, Nucl. Phys. B682 (2004) 487-520, [hep-th/0310252].

[34] N. Beisert, C. Kristjansen, and M. Staudacher, The Dilatation Operator of $\mathcal{N}=4$ Super Yang-Mills Theory, Nucl. Phys. B664 (2003) 131-184, [hep-th/0303060].

[35] G. C. Rossi, E. Sokatchev, and Y. S. Stanev, On the All-Order Perturbative Finiteness of the Deformed $\mathcal{N}=4$ Sym Theory, Nucl. Phys. B754 (2006) 329-350, [hep-th/0606284].

[36] D. I. Kazakov, Finite $\mathcal{N}=1$ SUSY Field Theories and Dimensional Regularization, Phys. Lett. B179 (1986) 352-354.

[37] D. I. Kazakov and L. V. Bork, Conformal Invariance $=$ Finiteness and Beta Deformed $\mathcal{N}=4$ Sym Theory, JHEP 08 (2007) 071, [arXiv:0706.4245].

[38] J. Ambjorn, R. A. Janik, and C. Kristjansen, Wrapping Interactions and a New Source of Corrections to the Spin-Chain / String Duality, Nucl. Phys. B736 (2006) 288-301, [hep-th/0510171].

[39] C. Sieg and A. Torrielli, Wrapping Interactions and the Genus Expansion of the 2- Point Function of Composite Operators, Nucl. Phys. B723 (2005) 3-32, [hep-th/0505071].

[40] Z. Bajnok and R. A. Janik, Four-Loop Perturbative Konishi from Strings and Finite Size Effects for Multiparticle States, Nucl. Phys. B807 (2009) 625-650, [arXiv:0807.0399].

[41] O. Aharony, B. Kol, and S. Yankielowicz, On Exactly Marginal Deformations of $\mathcal{N}=4$ Sym and Type IIB Supergravity on $\mathrm{AdS}_{5} \times S^{5}$, JHEP 06 (2002) 039, [hep-th/0205090].

[42] M. Kulaxizi, Marginal Deformations of $\mathcal{N}=4$ Sym and Open Vs. Closed String Parameters, hep-th/0612160.

[43] B. Sundborg, The Hagedorn Transition, Deconfinement and $\mathcal{N}=4$ Sym Theory, Nucl. Phys. B573 (2000) 349-363, [hep-th/9908001].

[44] O. Aharony, J. Marsano, S. Minwalla, K. Papadodimas, and M. Van Raamsdonk, The Hagedorn / Deconfinement Phase Transition in Weakly Coupled Large $N$ Gauge Theories, Adv. Theor. Math. Phys. 8 (2004) 603-696, [hep-th/0310285].

[45] O. Aharony, O. Bergman, D. L. Jafferis, and J. Maldacena, $\mathcal{N}=6$ Superconformal Chern-Simons-Matter Theories, M2-Branes and Their Gravity Duals, JHEP 10 (2008) 091, [arXiv: 0806.1218].

[46] O. Aharony, O. Bergman, and D. L. Jafferis, Fractional M2-Branes, JHEP 11 (2008) 043, [arXiv: 0807.4924].

[47] A. Gadde, E. Pomoni, and L. Rastelli, The Veneziano Limit of $\mathcal{N}=2$ Superconformal QCD: Towards the String Dual of $\mathcal{N}=2 S U\left(N_{c}\right)$ Sym with $N_{F}=2 N_{C}$, arXiv:0912.4918.

[48] A. Gadde, E. Pomoni, and L. Rastelli, Spin Chains in $\mathcal{N}=2$ Superconformal Theories: from the $Z_{2}$ Quiver to Superconformal QCD, arXiv:1006.0015.

[49] E. Pomoni and C. Sieg, From $\mathcal{N}=4$ Gauge Theory to $\mathcal{N}=2$ Conformal QCD: Three-Loop Mixing of Scalar Composite Operators, arXiv:1105.3487. 